\section{MS18-04 From tiny to tinier: combining techniques to reveal complex crystal intergrowths}

Serena C. Tarantino ${ }^{1,2}$, Alberto Zanetti ${ }^{1}$, Nobuyoshi Miyajiima ${ }^{3}$, Michele Zema ${ }^{2,4}$, Florian Heidelbach ${ }^{3}$, Paolo Ghigna ${ }^{5}$, Luca Olivi $^{6}$, Maurizio Mazzucchelli ${ }^{7}$

1. CNR-IGG, Pavia, Italy

2. Dept. of Earth and Environmental Sciences, University of Pavia, Italy

3. Bayerisches Geoinstitut, University of Bayreuth, Germany

4. International Union of Crystallography, Chester, UK

5. Dept. of Chemistry, University of Pavia, Italy

6. Elettra, Sincrotrone Trieste S.C.p.A., Italy

7. Dept. of Chemical and Geological Sciences, University of Modena and Reggio Emilia, Italy.

email: tarser06@unipv.it

A combined approach which makes use of long- and short-range crystallographic tools, such as optical, scanning and transmission electron microscopies, electron backscatter diffraction (EBSD), X-ray Absorption Spectroscopy (XAS), together with X-ray and electron diffraction has been used to characterize the highly unusual tight alternation of diopside and titanian pargasite lamellae. These lamellae have been observed in samples from spinel dunites belonging to the subcontinental mantle peridotite body of Balmuccia (Ivrea-Verbano Zone, Italy). The complicated multiphase arrangements displayed by most technological and geological materials on the one hand have dramatic effects on the physical properties of the material itself and, on the other hand, record its history. Microscopic inspections showed that in some grains, the thickness of the clinopyroxene lamellae $(\sim 20 \mu \mathrm{m})$ is around ten times that of amphibole, whereas in other cases the thicknesses of the two phases are comparable $(2-5 \mu \mathrm{m})$. EBSD maps revealed an extremely ordered phase separation, in which amphibole lamellae, as well as the late crystals, are all parallel to (010) in the pyroxene, as expected on the basis of crystallographic considerations and in agreement with TEM observations. Diffraction contrast images showed the presence of finer (less than $100 \mathrm{~nm}$ ) amphibole lamellae in the diopside, as well as the presence of semi-coherent interfaces giving rise to dislocations. Moreover, chain-width defects within the titanian pargasite have been revealed by HR-STEM images. STEM energy dispersive analyses revealed major element gradients across alternation of nm-scale lamellae, with diopside showing $\mathrm{Al}, \mathrm{Ti}, \mathrm{Na}$ and $\mathrm{Fe}$ increasing towards the contact with amphibole. Preliminary insights into the local structure of $\mathrm{Ti}^{4+}$, indicating a highly distorted octahedral coordination, have been obtained by means of XAS at the $\mathrm{Ti}-\mathrm{K}$ edge performed on the same crystals. On the basis of these observations, hypotheses on the origin of the tight alternation of diopside and titanian pargasite lamellae in such magmatic systems will be discussed.

Keywords: crystal intergrowth, diffraction, electron microscopy

\author{
MS18-05 Transmission electron microscopy \\ characterization of precipitate/matrix \\ interfaces in doped $\mathrm{Mn}_{4} \mathrm{Si}_{7}$ thermoelectric \\ crystal \\ Andrey Orekhov ${ }^{1}$, Elena Suvorova ${ }^{1}$
}

1. Shubnikov Institute of Crystallography of RAS, Moscow, Russia

email: andrey.orekhov@gmail.com

Higher manganese silicides (HMS) $\mathrm{MnSi}_{171-1.75}$ could attract more attention as potential middle-temperature thermoelectric materials that are cheap and non-toxic [1]. It was reported [2] that HMS crystals grown by Bridgman method with Ge, Mo and Al impurities had better properties $(\mathrm{ZT} \sim 0,7)$ as well the amount of $\mathrm{MnSi}$ precipitates significantly reduced. Impurities in the HMS crystal lattice are believed can contribute to increasing the electrical conductivity and decreasing the thermal conductivity, and thus to the improvement of the thermoelectric performance. This paper reports the results of electron microscopy study of Al, Ge, Mo doped HMS crystals with general formula of $\left(\left(\mathrm{Mn}_{0.98} \mathrm{Mo}_{0.02}\right)\left[\left(\mathrm{Si}_{0.98} \mathrm{Ge}_{0.02}\right)_{175}\right]_{0.99} \mathrm{Al}_{0.01}\right)$ grown by the Bridgman technique at $1473^{\circ} \mathrm{K}$ in Ar atmosphere. Microstructures and phase analysis of as grown samples were examined by SEM, EBSD and by HRTEM/HRSTEM. Chemical composition was estimated by energy dispersive X-ray spectroscopy (EDS) in both SEM and TEM. Electron diffraction patterns and EDS data showed the formation of tetragonal Mn4Si7 single crystals. No other Mn-Si phases with the stoichiometry of $\mathrm{MnSi}_{171-75}$ were observed. The crystal matrix contains $\mathrm{Al}$ and $\mathrm{Mo} \sim 0.5 \pm 0.3$ at $\%$ and $\mathrm{Ge} \sim 1.5 \pm 0.4$ at $\%$. No extra reflections on matrix crystal diffraction pattern were observed that means dopant elements occupy atom sites by substitution. Two types of nanosize precipitates in matrix crystal were found. Elliptical shape grains with 20x50 nm dimensions were arranges along [001] $\mathrm{Mn}_{4} \mathrm{Si}_{7}$. Another type precipitates were about $250 \mathrm{~nm}$ with strip-like structure. Nanodiffraction technique was applied for precipitates phase analysis. Hexagonal $\mathrm{Mn}_{5} \mathrm{Si}_{3}$ and cubic MnSi were observed for two types of precipitates. The structure of precipitates/matrix interface was examined by HRTEM. FFT filtering revealed extra planes at interfaces. The orientation relationships of precipitates/matrix were determined. The micro and nanostructure data of doped HMS may serve to optimize of crystal grows condition.

1. CRC Handbook of Thermoelectrics. Ed. D. M. Rowe (CRC Press, Boca Rayton, FL, 1995).

2. I. Aoyama, M.I. Fedorov, V.K. Zaitsev et al. // Jpn. J. Appl. Phys., 2005, V. 44, № 12 P. 8562-8570

Keywords: Structure of interfece, doped crystal, higher manganese silicide 